\title{
Year in Review: Synopsis of Selected Articles in Neuroanesthesia and Neurocritical Care from 2019
}

\author{
KokWeng Leong ${ }^{1}$ Tara Dalby ${ }^{1}$ Lashmi Venkatraghavan ${ }^{1}$ \\ ${ }^{1}$ Department of Anesthesia and Pain Medicine, Toronto Western \\ Hospital, University of Toronto, Toronto, Ontario, Canada
}

\begin{abstract}
Address for correspondence Lashmi Venkatraghavan, MBBS, MD, DNB, FRCA, FRCPC, Department of Anesthesia and Pain Medicine, Toronto Western Hospital, University of Toronto 2McL 405, Toronto Western Hospital, 399, Bathurst Street, Toronto, Ontario M5T 2S8, Canada (e-mail: Lashmi.Venkatraghavan@uhn.ca).
\end{abstract}
Abstract
Keywords
- key articles
- neurocritical care
- neurosurgery

This review is a synopsis of selected articles from neuroscience, neuroanesthesia, and neurocritical care from 2019 (January-November 2019). The journals reviewed included anesthesia journals, critical care medicine journals, neurosurgical journals, as well as high-impact medical journals such as the Lancet, Journal of American Medical Association, New England Journal of Medicine, and Stroke. This summary of important articles will serve to update the knowledge of anesthesiologists and other perioperative physicians who provide care to neurosurgical and neurocritical cases.

\section{Introduction}

\section{Reversal of Midazolam-Induced Motor Deficits by Fluma- zenil in Patients with Eloquent Area Glioma}

Sedation may unmask previously absent motor deficits, or exacerbate pre-existing motor deficits in patients with supratentorial brain tumor. Hence, it has the potential to cloud the neurological assessment of patients. However, the mechanism of these sedation-induced motor deficits and whether specific sedative antagonist can reverse the deficits, remain unknown. Lin et al studied 15 adult patients with eloquent area glioma scheduled for elective resection of the tumors. ${ }^{1}$ Prior to induction of anesthesia, motor coordination and eye-hand coordination of upper extremity (contralateral or ipsilateral to tumor location) were evaluated using Nine-Hole Peg Test in three phases: baseline (before midazolam), sedation (after midazolam), and reversal (after reversal of midazolam with flumazenil). Endpoint of midazolam administration was to achieve Observer Assessment of Alertness and Sedation score (OAAS) of 4 (i.e., sedated, but arousable and fully cooperative), and the endpoint of flumazenil reversal was to achieve OAAS score of 5 (i.e., fully alert). Study cohort was matched with control group and consisted of 17 patients with no brain lesions who presented for nonneurosurgery-related surgery. In the control group, dominant and nondominant extremities were considered instead of laterality to tumor location. The study showed that midazolam prolonged the time to completion of Nine-Hole Peg Test in both glioma patients (26.5 and 13.7 seconds slower in contralateral and ipsilateral hands, respectively, compared with baseline) and the control group (2.9 and 1.7 seconds slower in dominant hand and nondominant hands, respectively, compared with baseline). In patients with glioma, the effect of midazolam was not only confined to the contralateral extremities but also to the ipsilateral upper extremities, suggesting diaschisis and interruption in functional connectivity to remote undamaged area. In addition, patients with high-grade glioma took significantly longer to complete the test compared to those with low-grade glioma. This is probably because high-grade glioma is more aggressive and infiltrative, involving larger eloquent area that is unmasked at a greater degree by midazolam. Midazolam's effect was reversed completely after flumazenil in both groups. The authors concluded that despite "normal-looking" brain, these areas may already be affected by brain lesions, and are unmasked in some instances, such as sedated state. This study also suggested a gamma amino butyric acid-mediated mechanism to such observation.

\section{Risk Factors for Intraoperative Seizures during Elective Craniotomy}

Intraoperative seizure is an important catastrophic event with severe ramifications but the risk factors associated with intraoperative seizure are unknown. Kutteruf et al performed a single-center, retrospective review of medical records of 1,916 adult patients who underwent elective supratentorial 
craniotomy under total intravenous anesthesia with intraoperative neuromonitoring, with the aim of determining the perioperative risk factors for intraoperative seizure. ${ }^{2}$ Intraoperative seizures were identified from various electronic databases (anesthesia information management system, continuous quality improvement database, and intraoperative neuromonitoring database) along with the confirmation from both anesthesia and neurosurgical clinical notes as well as electroencephalogram (EEG) findings (when available). For each patient with intraoperative seizure, a control group consisting of randomly selected patients ( 1 case to 6 controls) who underwent elective supratentorial craniotomy in the same time frame, but did not develop intraoperative seizure was selected. Overall, incidence of intraoperative seizure was $2.3 \%$ $(43 / 1,916)$. Three factors were identified to be associated with increased risk of intraoperative seizures: history of preoperative seizure (odds ratio [OR] 2.18, 95\% confidence interval [CI] 1.07-4.46), brain tumor (OR 2.41, 95\% CI 1.16-4.19), and temporal craniotomy (OR 5.18, 95\% CI 2.03-13.25). Age, gender, brain trauma, preoperative use of anticonvulsants, presence of cerebral edema, presence of mass effect, lesion size, diagnosis other than brain tumor (aneurysm, arteriovenous malformation, and vascular occlusion) and surgical approaches other than temporal craniotomy were not associated with increased risk of intraoperative seizures. Prophylactic intraoperative use of phenytoin/fosphenytoin and levetiracetam were found to be protective against intraoperative seizure compared with no use of prophylactic anticonvulsants (phenytoin/fosphenytoin: OR, 0.09; 95\% CI, 0.03-0.29; $p<0.001$ and levetiracetam: OR, 0.34; 95\% CI, 0.14-0.87; $p=0.03$ ). When compared with each other, phenytoin/fosphenytoin was significantly more protective than levetiracetam (OR, 0.28; 95\% CI, 0.08-0.98; $p=0.046$ ).

\section{Perioperative Aspirin Use and Hemorrhagic Complications in Elective Craniotomy for Brain Tumor Surgery}

Increasing the number of patients taking aspirin (acetyl salicylic acid [ASA]) for primary prevention of cardiac and cerebral thromboembolic events often present with intracranial surgery. While ASA is commonly discontinued in such patients 7 to 10 days before any elective intracranial surgery, there are no strong data to support whether or not there is heightened risk of hemorrhagic complications associated with perioperative ASA continuation. Hanalioglu et al have conducted a retrospective study on 1,291 patients who underwent elective intracranial tumor surgery by a single surgeon over a 10-year period. ${ }^{3}$ They aimed to answer the following questions: (1) does the risk of hemorrhagic complications increase in patients who continue ASA use and (2) does the risk of thromboembolic complications increase in patients who have discontinued taking ASA? The patients were divided into three groups based on their perioperative ASA status: no ASA ( $n=1068)$; stopped ASA (low cardiovascular risk) $(n=104)$; and continued ASA (high cardiovascular risk) $(n=129)$. All the groups were similar in terms of patient demographics, tumor locations, pathologies, and extent of resections. Interestingly, intraoperative blood loss was not significantly different between the stopped $(186 \mathrm{~mL})$ and continued ( $220 \mathrm{~mL}$ ) ASA groups $(p=0.183)$. Most importantly, neither hemorrhagic $(0.6 \%, 0.9 \%$, and $0.8 \%$, respectively; $p=0.921)$ nor thromboembolic $(1.3 \%, 1.9 \%$, and $0.8 \%$; $p=0.779$ ) complication rates were significantly different between the groups, respectively. In summary, this study challenges the belief that ASA should be discontinued before all elective intracranial surgery, as there was no clear evidence for an increased rate of hemorrhagic complications with perioperative ASA use in intracranial tumor surgery. Therefore, in patients at high cardiovascular risk, ASA can be safely continued throughout the perioperative period for elective brain tumor surgery if potential benefits outweigh the risks of ASA continuation. However, the findings of the study are limited by the fact that it is a single center retrospective observational study, performed by just one surgeon, whose practices and skill level may not be generalizable.

\section{Mannitol in Critical Care and Neurosurgery}

Mannitol has been in clinical use for more than 50 years; yet, its clinical efficacy, impact on physiological parameters, and clinically relevant outcomes especially when compared with hypertonic saline (HTS) remain unclear. Zhang et al performed a systematic review and meta-analysis with the aim of studying various effects of mannitol namely intracranial pressure (ICP), cerebral perfusion pressure (CPP), mean arterial pressure (MAP), brain relaxation, serum sodium, and intraoperative fluid balance. ${ }^{4}$ In the setting of traumatic brain injury (TBI), the authors found that both mannitol and HTS are efficacious in reducing ICP, but HTS was more effective and associated with lower risk of ICP treatment failure (relative risk [RR]: 0.39, 95\% confidence interval [CI]: 0.18-0.81). Furthermore, both mannitol and HTS were effective in increasing CPP but there were no differences in post-treatment MAP between the two groups. Predictably, serum sodium was significantly lower and urine output was higher with mannitol use compared with HTS emphasizing the importance of monitoring urine output and intravascular volume status closely. In the setting of ischemic stroke, mannitol demonstrated no superiority over control in conscious level, motor capabilities, or sensory capabilities. However, in intracerebral hemorrhage, mannitol showed contradicting effects, with some randomized controlled trials (RCTs) demonstrating significant improvement while some RCTs showed no superiority over control. In elective craniotomy, both mannitol and HTS provided satisfactory brain relaxation, though HTS was, again, more effective in doing so. Mannitol also led to increased urine output and was associated with greater intravenous fluid replacement than HTS. However, despite the better brain relaxation profile of HTS compared with mannitol, no difference was demonstrated in length of intensive care unit (ICU) or overall hospital stay between mannitol and HTS. With regard to side effects, mannitol had a generally favorable safety profile, especially within the recommended dosage, but was associated with complications such as hyponatremia, hyperkalemia, pulmonary edema, 
acute renal failure, pulmonary embolism, hemodynamic disturbances, and compartment syndrome (secondary to extravasation).

\section{Validated Risk Score for Early Postoperative, Neurologic-Specific Complications after Elective Craniotomy for Brain Tumor Resection}

Craniotomy for brain tumor resection is associated with significant perioperative morbidity and mortality. However, routine admission to the ICU postoperatively is resource wasting, and has not been associated with improved outcomes. Cinotti et al have reviewed 45 perioperative data (in four categories-baseline characteristics, brain tumor data, perioperative data, and postoperative data) in 1,094 patients who presented for elective brain tumor resection, and developed a risk scoring (CranioScore) that predicts neurologic-specific complications in the first 24 hours after elective craniotomy. ${ }^{5}$ From this study cohort, eight high-risk factors were identified: Glasgow Coma Score (GCS) before surgery at or below 14 , history of brain tumor surgery, the greatest diameter of the tumor, cerebral midline shift at least $3 \mathrm{~mm}$, perioperative transfusion of blood products, maximum and minimal intraoperative systolic arterial pressure, and duration of surgery. These factors were then analyzed to form a composite scoring using the following formula.

CranioScore value $=-4.8094+[1.5149 \times$ (pre-operative Glasgow Coma Score) $]+[1.0534 \times$ (history of brain tumor surgery $)]+[0.00878 \times$ (greatest size of tumor in $\mathrm{mm})]+$ $[0.5114 \times($ midline shift $\geq 3 \mathrm{~mm})]+[0.5164 \times$ (transfusion of packed red blood cells and/or plasma and/or platelets)] + [0.0118 $\times($ maximum operative systolic arterial pressure $)]-$ $[0.0130 \times($ minimum operative systolic arterial pressure $)]+$ [0.2981 $\times$ (duration of surgery)]

The robustness of this scoring was subsequently validated externally in 830 patients from six ICUs. Severe complications occurred in $125(11.4 \%)$ and 90 (10.8\%) patients in the learning and validation cohorts, respectively. A composite score of $3 \%$ or below is associated with low risk of immediate postoperative complications, and hence, based on CranioScore, patient can be sent to general ward safely without increased risk of complications. While the negative predictive value of this scoring is high (100\%), the positive predictive value for immediate postoperative complication remains low (12.7\%). Furthermore, the complex calculation of CranioScore may limit its application in neurosurgical patients.

\section{Radiological Predictors of Difficult Direct Laryngoscopy in Patients with Acromegaly}

In patients with acromegaly, both bag mask ventilation and laryngoscopy can be very challenging and difficult. Conventional clinical predictors for difficult laryngoscopy, such as Mallampati score and upper lip bite test, have poor predictive values in these patients. Lee et al conducted a retrospective study on 90 patients with acromegaly who underwent trans-sphenoidal resection of pituitary adenoma, where
19 radiographic indices of airway anatomy from preoperative lateral skull X-ray (14 indices) and computed tomography (CT) images of ostiomeatal unit (OMU) (5 indices) were compared against Cormack-Lehane $(\mathrm{CL})$ grade at direct laryngoscopy. ${ }^{6}$ Difficult intubation was defined as CL grade III or IV. In this study, tongue area (TA) more than 2,600 $\mathrm{mm}^{2}$ (a measurement acquired from OMU CT), linear distance from alveolar line of mandible to hyoid bone (ALH) larger than $48 \mathrm{~mm}$ (measurement from skull X-ray), and linear distance from interior border of mandible to hyoid bone (MPH) more than $19.5 \mathrm{~mm}$ (measurement from skull X-ray) were the only indicators predictive of difficult direct laryngoscopy in patients with acromegaly. The negative predictive values (NPVs) of these indices were excellent, with values of $89 \%$, $94 \%$, and $87 \%$, respectively; however, the positive predictive values (PPVs) were poor, with values of $37 \%, 44 \%$, and $39 \%$, respectively. Interestingly, all these indices are measurements related to large tongue size, indicating that tongue size is a critical predictor for difficult direct laryngoscopy in patients with acromegaly. Other radiological indices of neck extension, submandibular space, prognathism, and epiglottis size were unrelated to difficult direct laryngoscopy. Of note, while older age was associated with difficult intubation but serum levels of growth hormone $(\mathrm{GH})$ or insulin-like growth factor-1 (IGF-1) were not associated with difficult intubation. This may be due to the fact that older age indicates longer duration of disease, while serum level of GH and IGF-1, which were measured only once preoperatively, did not reflect the duration of disease and the extent of hypertrophy.

\section{Impact of Different Intraoperative Fluid Administration Strategies during Spine Surgery}

Multilevel spine surgeries often involve large fluid shifts, significant blood loss, and hence major hemodynamic derangement. These patients are at risk of receiving a large volume of fluids, which is not ideal in a prolonged procedure in prone position leading to airway edema and respiratory compromise. This can result in delaying extubation, which inherently exposes the patients to risks associated with prolonged intubation. Ramachandran et al have done a single center, retrospective, observational study in 246 patients who underwent multilevel thoracolumbar spinal fusions involving four or more levels. ${ }^{7}$ The aim of this study was to determine if the use of predominantly crystalloid versus colloid fluid was positively associated with delayed extubation. The authors divided the cohort into groups: delayed extubation (DEX) or immediate extubation (IMEX), and performed propensity-matched analysis to determine the likelihood of fluid type, volume and ratios on the outcome. Contrary to their hypothesis, total fluid administration in the two groups was not significantly different; however, further subdivision showed that the crystalloid to colloid ratio was higher in the DEX group $8.5 \pm 6.4$ versus $6.8 \pm 4.0 ; p=0.03$. In this patient group, DEX was associated with increased ICU, hospital stay, and complications. Furthermore, they divided the patients into three groups by estimated blood loss (EBL), 
with group EBL > 30\%, more likely to be elderly, exposed to greater surgical invasiveness, and a higher percentage of delayed extubation. They also divided the patients into groups according to crystalloid to colloid ratio $<3: 1$ or $>3: 1$, based on the assumption that three times as much crystalloid is required to increase the intravascular space. This analysis showed that $26 \%$ of patients receiving crystalloid to colloid ratio $>3: 1$ had DEX compared with none of those receiving crystalloid to colloid ratio $\leq 3: 1(p=0.009)$. The authors conclude that overzealous crystalloid infusion is a risk factor for delayed extubation in these surgical cases and, colloids are better replacement fluid with increasing blood loss. However, details regarding the decision behind delayed versus immediate extubation are not clear as there may be multiple confounders behind the decision-making process.

\section{Incidence of False-Positive and False-Negative Changes in Evoked Potential Monitoring during Clipping of Unruptured Intracranial Aneurysms}

In patients undergoing clipping of unruptured intracranial aneurysm (UIA), intraoperative neurophysiological monitoring such as somatosensory evoked potential (SSEP) and motor evoked potential (MEP) are often utilized to detect intraoperative cerebral ischemia and to prevent postoperative neurological deficits. Sensitivity and specificity of SSEP/ MEP have been evaluated in previous studies, but incidences of false positive and false negative were unknown. Chung et al analyzed medical records of 1,514 patients with UIA with no baseline neurological deficits, who underwent microsurgery clipping of aneurysm with intraoperative SSEP and MEP monitoring. ${ }^{8}$ Motor power examination was performed in the immediate postoperative period, and these findings were correlated to the presence (or absence) of significant changes (defined as more than $50 \%$ decrease in amplitude) in evoked potential (EP) monitoring during surgery. False negative was defined as new onset motor deficits detected in the immediate postoperative period due to a newly developed brain lesion identified from brain imaging, but without significant EP changes during surgery, while false positive was defined as any significant EP changes that were persistent until completion of EP monitoring in spite of all corrective efforts during surgery with no postoperative motor deficits. This study showed that false-positive and false-negative rates were low $(0.09 \%$ and $0.53 \%$, respectively). Specificity and negative predictive values were high (0.941-0.995 and 0.993-0.994, respectively), but sensitivity and positive predictive value were low (0.000-0.100 and 0.000-0.011, respectively). Due to the small number of false positive and false negative in this study, subanalysis using various indicators, such as age, gender, location of aneurysm, size of aneurysm, and number of aneurysms, to determine whether any group would benefit more from EP monitoring, did not demonstrate any significant findings.

\section{Effect of Different Intraoperative Blood Pressure and End-Tidal Carbon Dioxide Threshold during Aneurysm Occlusion on Neurological Outcome}

Aneurysmal subarachnoid hemorrhage is associated with disruption of cerebral autoregulation, with resultant pressure-dependent cerebral blood flow. Furthermore, hypocapnia has been shown to be associated with poor outcomes in traumatic brain injury, while hypercapnia improves outcome in general surgical patients. To date, no large studies have investigated the effect of different blood pressure targets and arterial carbon dioxide tensions $\left(\mathrm{PaCO}_{2}\right)$ specific to general anesthesia during the treatment of ruptured aneurysm. Akkermans et al conducted a retrospective observational study on 1,099 patients who underwent general anesthesia for obliteration of ruptured intracranial aneurysm (neurosurgical clipping or endovascular coiling), where time-weighted average of end-tidal carbon dioxide $\left(\mathrm{ETCO}_{2}\right)$ and mean arterial pressure (MAP) were recorded and analyzed for their relationship to neurological outcome (Glasgow Outcome Scale [GOS] at discharge and at 3 months). ${ }^{9}$ For $\mathrm{ETCO}_{2}$, four thresholds were defined: less than $30 \mathrm{mmHg}$, less than $35 \mathrm{mmHg}$, less than $40 \mathrm{mmHg}$, and less than $45 \mathrm{mmHg}$. while for MAP, the following threshold were defined: $<60 \mathrm{mmHg},<70 \mathrm{mmHg},<80 \mathrm{mmHg},>90 \mathrm{mmHg}$, and $>100 \mathrm{mmHg}$. Interestingly, the authors found that there was no association between any of the time-weighted average area under the curve threshold for $\mathrm{ETCO}_{2}$ or MAP on GOS on discharge or at 3 months, and this relationship was valid even after adjusting for potential confounders. These findings were also robust irrespective of preoperative SAH grading, treatment modalities, (clipping or coiling), or timing of treatment. This finding was also relevant for extreme values of hypocapnia $\left(\mathrm{ETCO}_{2}\right.$ less than $30 \mathrm{mmHg}$ ) or hypotension (MAP $<60 \mathrm{mmHg}$ ). One limitation of this study was the assumption that $\mathrm{ETCO}_{2}$ bears a close and fixed relationship to $\mathrm{PaCO}_{2}$.

\section{Comparative Efficacy of Intra-arterial Plus Intravenous Milrinone Versus Intravenous Milrinone Protocols for the Treatment of Cerebral Vasospasm Following Subarachnoid Hemorrhage}

Milrinone, a direct vasodilator owing to its selective inhibition of phosphodiesterase-3, has emerged as an option to reverse angiographically confirmed cerebral vasospasm. Earlier protocol involved selective infusion of milrinone into major artery supplying vasospastic segment followed by intravenous infusion; later, intravenous (IV) regime without intra-arterial milrinone has also been advocated. Comparative effectiveness of either regime was unknown. Crespy et al, retrospectively, reviewed medical records of 101 patients who received either intra-arterial + intravenous (IA + IV) regime (22 patients) or IV regime (77 patients). In the IA + IV group, under general anesthesia, $8 \mathrm{mg}$ of milrinone was 
infused over 30 minutes into the main artery supplying the vasospastic segment in the neuroangio suite..$^{10}$ If there was incomplete reversal of vasospasm, IA milrinone was repeated up to maximum of $24 \mathrm{mg}$. Following this, patients received continuous IV infusion of milrinone $1 \mu \mathrm{g} / \mathrm{kg} / \mathrm{min}$ until 14 days after the initial bleed. The IV protocol was simpler, where milrinone was infused intravenously at bedside at $1 \mu \mathrm{g} / \mathrm{kg} / \mathrm{min}$ for 7 days, followed by neurovascular imaging. If there was radiological improvement, the milrinone was stopped within 48 hours; if there was deterioration, patient was subjected to intra-arterial procedure; and if there was clinical improvement only, IV milrinone was continued for another 7 days. The primary endpoint was reversion rate of vasospastic arterial segment (defined as narrowing $<50 \%$ ) following the first IA infusion (in the IA + IV group) compared with reversion rate 7 days after IV infusion (in the IV group). The authors found that there was no difference in proportion of patients requiring rescue procedures, neurological outcome, mortality in both groups, or rate of milrinone-induced hypotension. IV infusion was as efficient as combined IA + IV, and avoided certain risks associated with IA milrinone including general anesthesia, insertion of microcatheter, stroke, or adverse event associated with transport to neuroangio suite. One important caveat of note is that reversion of vasospasm should not be interpreted to equate with better clinical outcome, as many aspects of microvascular dysfunction other than vessel narrowing, such as endothelial damage, thrombosis, inflammation, or cortical spreading depolarization, have been found to play a role in delayed cerebral ischemia.

\section{Spatial and Temporal Pattern of Ischemia and Abnormal Vascular Function Following Traumatic Brain Injury}

Secondary brain insults, such as ischemia, intracranial hypertension, and cerebral edema, contribute significantly to morbidity and mortality following traumatic brain injury (TBI). Changes in cerebrovascular physiology after TBI contribute to these secondary insults. Adequate delivery of oxygen and substrate by optimizing CPP, and controlling ICP are the mainstay in the management of these patients. Launey et al have conducted a single-center prospective observational cohort study with data collected over a long time period (February 1998 to July 2014) in 68 patients with moderate to severe TBI and 27 controls (healthy volunteers). ${ }^{11}$ The aim of this study was to investigate derangement in cerebral physiology following TBI using 15oxygen positron emission tomography (PET) in a large patient cohort up to 10 days after TBI. Patient care was standardized and protocolized aiming for ICP less than $20 \mathrm{mmHg}$, CPP 60 to $70 \mathrm{mmHg}$, with continuous monitoring with brain tissue oximetry $\left(\mathrm{BTPO}_{2}\right)$ and jugular venous oxygen saturations $\left(\mathrm{SJVO}_{2}\right)$. The PET studies showed reduced cerebral blood flow (CBF) and cerebral metabolic requirement for oxygen $\left(\mathrm{CMRO}_{2}\right)$ in patients with TBI at all time points. Early ischemia ( $<24$ hours) occurs in approximately two-thirds of patients, was detectable up to 10 days post injury. Ischemia was not limited to patients with intracranial hypertension and it was inconsistently detected by jugular or brain tissue oximetry. There was substantial pathophysiological heterogeneity between patient and within-patient with both ischemia and hyperemia commonly coexist, possibly reflecting abnormalities in flow-metabolism coupling. Increased CBV may contribute to intracranial hypertension but can coexist with ischemia. These results emphasize the need to consider cerebrovascular pathophysiological complexity when managing patients with TBI. Pathophysiologic heterogeneity indicates that bedside physiological monitoring with devices that measure global (jugular venous saturation) or focal (tissue oximetry) brain oxygenation should be interpreted with caution.

\section{Effect of Early Sustained Prophylactic Hypothermia on Neurological Outcomes Among Patients with Severe Traumatic Brain Injury: The POLAR-RCT}

Mild hypothermia has long been hypothesized to provide neuroprotection through inhibiting cerebral inflammation and slowing of biochemical processes leading to neurotoxicity. Definitive clinical data remain elusive on this issue. While one randomized controlled trial showed no benefit, a recent meta-analysis showed a reduced mortality with mild hypothermia in patients with TBI. ${ }^{12,13}$ Cooper et al conducted a multicenter international randomized controlled trial; patients with severe TBI (initial GCS $>9$ or intubated, aged 18-60) were randomized to hypothermia $(n=256)$ or normothermia groups $(n=255) .^{14}$ The hypothermia group received $2,000 \mathrm{~mL}$ of ice-cold saline $\left(4^{\circ} \mathrm{C}\right)$ to reduce the core temperature to $35^{\circ} \mathrm{C}$. In addition, in patients randomized to hypothermia if deemed not at great risk of hemorrhagic complications, the temperature was further reduced to $33^{\circ} \mathrm{C}$ using surface cooling and maintained for at least 72 hours or up to 7 days if high intracranial pressure was sustained. The outcomes assessed were favorable neurological outcome, measured by the Glasgow Outcome Scale-Extended (GOS-E) score of 5 to 8, or independent living at 6 months. The study showed no significant difference in neurological outcomes between the groups. It is important to note that the median time to reach temperature of $33^{\circ} \mathrm{C}$ was 10 hours, due to the necessity to rule out other injuries. Many patients assigned intervention (32\%) did not ever reach the target temperature or were withdrawn early due to clinical decisions. In addition, three patients in the hypothermia group developed propofol infusion syndrome due to reduced hepatic metabolism of propofol.

\section{Extending Thrombolysis to 4.5 to 9 Hours Using Perfusion Imaging: a Systematic Review and Meta-Analysis of Individual Patient Data}

Traditionally, thrombolysis is recommended only to patients presenting within 4.5 hours of onset of acute ischemic stroke, thus excluding a large number of patients who may benefit from thrombolysis. Several recent studies have 
investigated extending clinical indications for thrombolysis from 4.5 to 9 hours after stroke onset. Campbell et al have done a systematic review and meta-analysis of randomized trials published between January 1, 2006, and March 1, $2019 .{ }^{15}$ The study included trials where adults ( $>18$ years of age) with acute ischemic stroke treated with alteplase or placebo after 4.5 hours of stroke onset or who presented with wake-up stroke. In addition, all patients needed to have imaging with magnetic resonance imaging (MRI) or CT perfusion. Outcomes of interest were excellent functional outcomes at 3 months, modified Rankin Scale (mRS) 0 to 1 , adjusted for both age and clinical severity. There were a total of three trials included-the EXTEND, ECASS 4-EXTEND, and EPITHET with the total of 414 patients, 213 receiving alteplase and 201 received placebo. Excellent functional outcomes were achieved in $36 \%$ patients ( 76 out of 211 ) in the alteplase group compared with $28 \%$ patients in the placebo group (58 out of 199) (adjusted OR: 1.86, 95\% CI: $1.15-2.99 ; p=0.11)$. Symptomatic intracranial hemorrhage was more common in the thrombolysis group (5\%) than the placebo group $(<1 \%)$ but the difference was not statistically significant. Similarly, more deaths occurred in the intervention group (13\% vs. $11 \%$ ) and again this did not reach statistical significance. Hence, emerging evidence indicates that patients with potentially salvageable brain tissue on perfusion imaging presenting outside the current time limits for thrombolysis will likely benefit from thrombolysis treatment.

\section{Association between Time to Treatment with Endovascular Reperfusion Therapy and Outcomes}

Re-establishment of perfusion to ischemic brain tissue in a timely manner is a priority in the management of acute ischemic stroke. However, the extent to which time influences outcome and generalizability to routine clinical practice remains uncertain. Jahan et al conducted a large retrospective cohort study to determine the association between the time to endovascular thrombectomy (ET) and outcome. Data were collected from a United States-wide registry of patients who had endovascular treatment for acute ischemic stroke from multiple centers, accounting for differences in clinical practice. ${ }^{16}$ The data included 6,756 patients who had ET within 8 hours of stroke onset. The stroke onset time was taken as the time the patient was last seen well. Main clinical outcomes included discharge to home, independent ambulation at discharge, freedom from disability (mRS score $0-1$ ) at discharge, functional independence (mRS score $0-2$ ) at discharge, and spontaneous intracerebral hemorrhage (sICH). Two time intervals were evaluated for relation to each of these outcomes: (1) onset (last-known well) to arterial puncture and (2) hospital arrival to arterial puncture (door-to puncture time). The median onset to puncture time was 230 minutes and hospital arrival to puncture time was
87 minutes. Good reperfusion occurred in $85.9 \%$ of patients in this study cohort. Importantly, decreasing stroke onset to puncture time could modify this outcome. There is a nonlinear association between stroke onset and reperfusion time, with steeper slopes between 30 and 270 minutes than between 271 and 480 minutes. In the 30- to 270 -minute time frame, faster onset to puncture in 15 -minute increments was associated with higher likelihood of achieving independent ambulation at discharge, lower in-hospital mortality/hospice discharge, and lower risk of sICH. Faster door-to-puncture times were similarly associated with improved outcomes, including in the 30- to 120-minute window. Every 15-minute delay was found to have significant effect on disability-free outcome. Thus, this is a very important study contributing to our understanding of the urgent and time-sensitive nature of providing ET.

\section{Decreases in Blood Pressure during Thrombectomy Are Associated with Larger Infarct Volumes and Worse Functional Outcomes}

Hypotension in patients with acute ischemic stroke may lead to further extension of the infarct size due to the reduction in blood flow to the vulnerable ischemic penumbra. Peterson et al have looked at the association between hypotension during endovascular thrombectomy (ET), final infarct size, and functional outcome. ${ }^{17}$ This was a retrospective cohort study of 319 patients from two major stroke centers. Blood pressure values from either arterial line or noninvasive blood pressure measurements during ET were retrieved from electronic records. Change in MAP was calculated as the difference between admission MAP and lowest MAP during endovascular thrombectomy until recanalization. Sustained hypotension was measured as the area between admission MAP and continuous measurements of intraprocedural MAP (aMAP). Final infarct volume was measured using MRI at 24 hours, and functional outcome was assessed using the mRS at discharge and 90 days. Associations with outcome were analyzed using linear and ordinal multivariable logistic regression. A large number of patients experienced hypotension during ET (87\%), with a mean reduction in MAP $31 \pm 20 \mathrm{mmHg}$. The change in MAP correlated with infarct growth on perfusion imaging $(p=0.036)$ and final infarct volume ( $p=0.035)$. Greater reduction in MAP was associated with poor functional outcome at discharge (adjusted OR for every $10 \mathrm{mmHg}$ : $1.17 ; 95 \% \mathrm{CI}: 1.04-1.32 ; p=0.009$ ), and at 90 days (adjusted OR for every $10 \mathrm{mmHg}: 1.22$; $95 \%$ CI: $1.07-$ 1.38; $p=0.003$ ). One limitation of using noninvasive blood pressure measurement was that the periods of hypotension between the cuff cycling times (3-5 minutes) was often missed. This study suggests that extension of infarct volume is the mechanism for the dose-dependent association between intraprocedural hypotension and worse functional outcomes after ET. 


\section{Hemodynamic Management of Patients Undergoing Endovascular Treatment for Acute Ischemic Stroke Under Conscious Sedation}

Hemodynamic instability is common in patients undergoing endovascular thrombectomy (ET) for acute ischemic stroke (AIS) regardless of the anesthetic technique. Though hemodynamic instability under general anesthesia is well documented, very limited information is available in the literature regarding the incidence and the magnitude of hemodynamic instability during mechanical thrombectomy under conscious sedation. Alcaraz et al have reviewed the hemodynamic intervention during ET for AIS in a single center retrospective study of 126 patients over a period of 4 years. ${ }^{18}$ All patients received conscious sedation (92\%) or no sedation (8\%). Primary outcome was hemodynamic intervention, defined as administration of vasoactive drugs to maintain systolic blood pressure (SBP) between 140 and $180 \mathrm{mmHg}$. The secondary outcome was poor hemodynamic control, defined as SBP outside target for > 15 minutes despite hemodynamic intervention. Hemodynamic interventions were frequent, $77.8 \%$ of patients experienced hypotension and 19.5\% hypertension at least once during the treatment. Poor hemodynamic control occurred in $12.7 \%$. A lower baseline systolic blood pressure was found to be a risk factor for intraprocedural hypotension OR: 0.92 (95\% CI: 0.89-0.96; $p=0.001)$. The study findings suggest that the routine presence of anesthesiologists during ET may be helpful in maintaining hemodynamic stability and allowing rapid treatment of emergent complications. In addition, an individualized approach with tailored hemodynamic targets is required during management of patients undergoing ET for AIS.

\section{Blood Pressure and Outcome after Mechanical Thrombectomy with Successful Revascularization}

Hypertension is common after an acute ischemic stroke. Current guidelines recommend systolic blood pressure (SBP) $<180 \mathrm{mmHg}$ following reperfusion to avoid hemorrhagic complications and to minimize the reperfusion injury. Anadani et al examined the relationship between admission SBP, SBP during the first 24 hours after revascularization, and their correlation with functional neurological outcome and complications. ${ }^{19}$ This retrospective observational study was conducted across 10 stroke centers around the United Kingdom and Europe and included 1,245 patients with anterior circulation ischemic stroke who had successful revascularization. Primary outcome was functional independence at 90 days using the mRS either face-to-face interviews in follow-up clinics or telephone interviews; $49 \%$ patients had good functional outcome ( $\mathrm{mRS} 0-2$ ). The incidence of spontaneous intracerebral hemorrhage and malignant cerebral edema requiring hemicraniectomy were $4.7 \%$ and $4 \%$, respectively. Higher admission SBP was not associated with a poor functional outcome, but conferred higher risk of hemorrhagic complications. However, there was a significant association between the 24-hour postrevascularization SBP parameters and functional outcome and complications. Patients in poor outcome group had higher mean SBP (131 \pm 16 vs. $127 \pm 14 \mathrm{mmHg}, p<0.001)$, maximum SBP $(164 \pm 25$ vs. $157 \pm 24, p<0.001$ ), and higher SBP range (62 \pm 26 vs. $56 \pm 24$, $p<0.001$ ) when compared with good outcome group. However, the authors do acknowledge that the BP measurements were not standardized in this study.

\section{Narrative Reviews of Interest}

Several excellent review articles focused on topics of particular interest to the neuroanesthesiologists were published over the last year. Hindman has done a two-part review to provide a practical perspective on the clinical literature regarding anesthesia care of patients with acute ischemic stroke treated with endovascular thrombectomy. ${ }^{20,21}$ The first part reviews the development of endovascular thrombectomy and the determinants of endovascular thrombectomy effectiveness irrespective of method of anesthesia. ${ }^{20}$ The second part provides a pragmatic approach to anesthesia decision making and management of patients treated with endovascular thrombectomy. ${ }^{21}$ Porter and Dinsmore have reviewed the current evidence pertaining to the perioperative management of patients on direct oral anticoagulants presenting for elective and emergency intracranial surgery. ${ }^{22}$ The use of direct oral anticoagulants is increasing rapidly, because of perceived benefits over older agents, such as predictable pharmacokinetics and a reduced risk of bleeding. This review highlights emerging therapies, including specific antidotes, as well as areas where the evidence base is likely to improve in the future. Thorup et al have done a comprehensive narrative review on the effects of vasopressors on cerebral circulation and oxygenation in health and in patients with traumatic brain injury. ${ }^{23}$ Other reviews of interest include a review on cerebral intraparenchymal hemorrhage by Gross et $\mathrm{al}^{24}$ and a narrative review of cardiovascular abnormalities after spontaneous intracerebral hemorrhage by Lee et al. ${ }^{25}$ Finally, there is a recent update on the practice advisory for perioperative visual loss associated with spine surgery by the American Society of Anesthesiologists Task Force on Perioperative Visual Loss. ${ }^{26}$

\section{Conflict of Interest}

None declared.

\section{References}

1 Lin N, Han R, Hui X, Zhang K, Gelb AW. Midazolam sedation induces upper limb coordination deficits that are reversed by flumazenil in patients with eloquent area gliomas. Anesthesiology 2019;131(1):36-45

2 Kutteruf R, Yang JT, Hecker JG, Kinney GA, Furman MA, Sharma D. Incidence and risk factors for intraoperative seizures during elective craniotomy. J Neurosurg Anesthesiol 2019;31(2):234-240

3 Hanalioglu S, Sahin B, Sahin OS, et al. Effect of perioperative aspirin use on hemorrhagic complications in elective craniotomy for brain tumors: results of a single-center, retrospective cohort study. J Neurosurg 2019; doi 10.3171/2018.12. JNS182483 
4 Zhang W, Neal J, Lin L, et al. Mannitol in critical care and surgery over 50+ years: a systematic review of randomized controlled trials and complications with meta-analysis. J Neurosurg Anesthesiol 2019;31(3):273-284

5 Cinotti R, Bruder N, Srairi $M$, et al. Société Française d'Anesthésie-Réanimation (SFAR) Research Network. Prediction score for postoperative neurologic complications after brain tumor craniotomy: a multicenter observational study. Anesthesiology 2018;129(6):1111-1120

6 Lee HC, Kim MK, Kim YH, Park HP. Radiographic predictors of difficult laryngoscopy in acromegaly patients. J Neurosurg Anesthesiol 2019;31(1):50-56

7 Ramchandran S, Day LM, Line B, et al. The impact of different intraoperative fluid administration strategies on postoperative extubation following multilevel thoracic and lumbar spine surgery: a propensity score matched analysis. Neurosurgery 2019;85(1):31-40

8 Chung J, Park W, Hong SH, et al. Intraoperative use of transcranial motor/sensory evoked potential monitoring in the clipping of intracranial aneurysms: evaluation of false-positive and false-negative cases. J Neurosurg 2018;130(3):936-948

9 Akkermans A, van Waes JA, Peelen LM, Rinkel GJ, van Klei WA. Blood pressure and end-tidal carbon dioxide ranges during aneurysm occlusion and neurologic outcome after an aneurysmal subarachnoid hemorrhage. Anesthesiology 2019;130(1):92-105

10 Crespy T, Heintzelmann M, Chiron C, et al. Which protocol for milrinone to treat cerebral vasospasm associated with subarachnoid hemorrhage? J Neurosurg Anesthesiol 2019;31(3):323-329

11 Launey Y, Fryer TD, Hong YT, et al. Spatial and temporal pattern of ischemia and abnormal vascular function following traumatic brain injury. JAMA Neurol 2019; doi 10.1001/ jamaneurol.2019.3854

12 Clifton GL, Miller ER, Choi SC, et al. Lack of effect of induction of hypothermia after acute brain injury. $\mathrm{N}$ Engl J Med 2001;344(8):556-563

13 Olah E, Poto L, Hegyi P, et al. Therapeutic whole-body hypothermia reduces death in severe traumatic brain injury if the cooling index is sufficiently high: meta-analyses of the effect of single cooling parameters and their integrated measure. J Neurotrauma 2018;35(20):2407-2417

14 Cooper DJ, Nichol AD, Bailey M, et al. POLAR Trial Investigators and the ANZICS Clinical Trials Group. Effect of early sustained prophylactic hypothermia on neurologic outcomes among patients with severe traumatic brain injury: the POLAR randomized clinical trial. JAMA 2018;320(21):2211-2220

15 Campbell BCV, Ma H, Ringlet PA, et al. EXTEND, ECASS-4, and EPITHET Investigators. Extending thrombolysis to 4.5-9 h and wake-up stroke using perfusion imaging: a systematic review and meta-analysis of individual patient data. Lancet 2019;394:139-147

16 Jahan R, Saver JL, Schwamm LH, et al. Association between time to treatment with endovascular reperfusion therapy and outcomes in patients with acute ischemic stroke treated in clinical practice. JAMA 2019;322(3):252-263

17 Petersen NH, Ortega-Gutierrez S, Wang A, et al. Decreases in blood pressure during thrombectomy are associated with larger infarct volumes and worse functional outcome. Stroke 2019;50(7):1797-1804

18 Alcaraz G, Chui J, Schaafsma J, et al. Hemodynamic management of patients during endovascular treatment of acute ischemic stroke under conscious sedation: a retrospective cohort study. J Neurosurg Anesthesiol 2019;31(3):299-305

19 Anadani M, Orabi MY, Alawieh A, et al. Blood pressure and outcome after mechanical thrombectomy with successful revascularization. Stroke 2019;50(9):2448-2454

20 Hindman BJ. Anesthetic management of emergency endovascular thrombectomy for acute ischemic stroke, part 1: patient characteristics, determinants of effectiveness, and effect of blood pressure on outcome. Anesth Analg 2019;128(4):695-705

21 Hindman BJ, Dexter F. Anesthetic management of emergency endovascular thrombectomy for acute ischemic stroke, part 2: integrating and applying observational reports and randomized clinical trials. Anesth Analg 2019;128(4):706-717

22 Porter J, Dinsmore J. Perioperative management of direct oral anticoagulants in intracranial surgery. J Neurosurg Anesthesiol 2019. doi:10.1097/ANA.0000000000000629

23 Thorup L, Koch KU, Upton RN, Østergaard L, Rasmussen M. Effects of vasopressors on cerebral circulation and oxygenation: a narrative review of pharmacodynamics in health and traumatic brain Injury. J Neurosurg Anesthesiol 2019. doi: 10.1097/ANA.0000000000000596

24 Gross BA, Jankowitz BT, Friedlander RM. Cerebral intraparenchymal hemorrhage: a review.JAMA 2019;321(13):1295-1303

25 Lele A, Lakireddy V, Gorbachov S, Chaikittisilpa N, Krishnamoorthy V, Vavilala MS. A narrative review of cardiovascular abnormalities after spontaneous intracerebral hemorrhage. J Neurosurg Anesthesiol 2019;31(2):199-211

26 Practice Advisory for Perioperative Visual Loss Associated with Spine Surgery. Practice advisory for perioperative visual loss associated with spine surgery 2019: an updated report by the American Society of Anesthesiologists Task Force on Perioperative Visual Loss, the North American Neuro-Ophthalmology Society, and the Society for Neuroscience in Anesthesiology and Critical Care. Anesthesiology 2019;130(1):12-30 